\title{
Media multitasking and memory: Differences in working memory and long-term memory
}

\author{
Melina R. Uncapher ${ }^{1,3}$ - Monica K. Thieu ${ }^{1,3}$ - Anthony D. Wagner ${ }^{1,2,3}$
}

Published online: 30 July 2015

(C) Psychonomic Society, Inc. 2015

\begin{abstract}
Increasing access to media in the 21st century has led to a rapid rise in the prevalence of media multitasking (simultaneous use of multiple media streams). Such behavior is associated with various cognitive differences, such as difficulty filtering distracting information and increased trait impulsivity. Given the rise in media multitasking by children, adolescents, and adults, a full understanding of the cognitive profile of media multitaskers is imperative. Here we investigated the relationship between chronic media multitasking and working memory (WM) and long-term memory (LTM) performance. Four key findings are reported (1) heavy media multitaskers (HMMs) exhibited lower WM performance, regardless of whether external distraction was present or absent; (2) lower performance on multiple WM tasks predicted lower LTM performance; (3) media multitasking-related differences in memory reflected differences in discriminability rather than decision bias; and (4) attentional impulsivity correlated with media multitasking behavior and reduced WM performance. These findings suggest that chronic media multitasking is associated with a wider attentional scope/higher attentional impulsivity, which may allow goal-irrelevant information to compete with goal-relevant information. As a consequence,
\end{abstract}

Electronic supplementary material The online version of this article (doi:10.3758/s13423-015-0907-3) contains supplementary material, which is available to authorized users.

Melina R. Uncapher

melina.u@stanford.edu

1 Department of Psychology, Stanford University, Stanford, CA 94305, USA

2 Neurosciences Program, Stanford University, Stanford, CA 94305, USA

3 Jordan Hall, Bldg 420, Stanford, CA 94305-2130, USA heavy media multitaskers are able to hold fewer or less precise goal-relevant representations in WM. HMMs' wider attentional scope, combined with their diminished WM performance, propagates forward to yield lower LTM performance. As such, chronic media multitasking is associated with a reduced ability to draw on the past - be it very recent or more remote - to inform present behavior.

Keywords Episodic memory $\cdot$ Attention $\cdot$ Distractor filtering $\cdot$ Impulsivity $\cdot$ Signal detection theory

In a world that affords ubiquitous access to information, many people often multitask with multiple streams of media. The rapid rise in "media multitasking" (Rideout, Foehr, \& Roberts, 2010) has generated considerable scientific and societal interest in the relationship between this behavior and fundamental aspects of human cognition. Initial studies have examined aspects of cognitive control, finding that heavy media multitaskers (HMMs) perform poorly in tasks involving working memory (Minear, Brasher, McCurdy, Lewis, \& Younggren, 2013) and distractor filtering (Cain \& Mitroff, 2011; Ophir, Nass, \& Wagner, 2009), with variable effects on task switching (c.f. Alzahabi \& Becker, 2013; Minear et al., 2013; Ophir et al., 2009). Other studies have examined the relationship between media multitasking behavior and psychosocial variables such as trait impulsivity (Minear et al., 2013; Sanbonmatsu, Strayer, Medeiros-Ward, \& Watson, 2013; Shih, 2013). In general, greater self-reported media multitasking appears associated with higher self-reported measures of impulsiveness, either on Attention (Sanbonmatsu et al., 2013) or Motor subscales (Minear et al., 2013; Sanbonmatsu et al., 2013; c.f. Shih, 2013).

While the direction of causality is unknown - whether frequent media multitasking induces psychosocial and cognitive 
control differences or whether people with these differences gravitate toward more frequent media multitasking - the initial observations demand a deeper understanding of the cognitive costs (and benefits) associated with frequent media multitasking. This is especially urgent given that more and more young people, whose brains are still developing, are engaging in media multitasking (Rideout et al., 2010).

Progress may come from a fuller investigation of how cognitive performance varies as a function of media multitasking behavior. For instance, while the aforementioned studies point to working memory (WM) differences, the conditions in which such differences are obtained remain underspecified. Using a signal detection decision-making framework, WM performance can be characterized by a discriminability parameter $\left(d^{\prime}\right)$ that indexes the precision or amount of information held in WM, and a bias parameter $(C)$ that indexes the propensity to endorse that a signal was detected (Green \& Swets, 1966). Given that HMMs demonstrate higher trait impulsivity (Minear et al., 2013; Sanbonmatsu et al., 2013), it remains an open question as to whether this population may require less evidence to reach a decision, which would manifest as a more liberal response bias when making WM judgments. Moreover, HMMs' greater sensitivity to internal and external distraction may manifest as reduced WM performance even in the absence of external distractors.

A second line of open questions concerns whether the WM performance differences in HMMs have consequences for long-term memory (LTM). To date, investigations of media multitasking have focused on cognition directed to the present moment/very recent past or series of moments (e.g., visual working memory; n-back; task switching). It remains unknown whether the impairments in moment-bymoment cognition observed in HMMs have consequences for future cognition that depends on long-term memories for those moments.

To address these open questions, we measured discrimination and bias during WM performance, and then related these measures to corresponding measures during LTM performance (including measures of LTM for information encountered in one of the WM tasks) in a large sample of participants $(N=143)$.

\section{Method}

\section{Participants}

We recruited 143 participants (83 females; $18-35$ years old, mean $=22.1$ years, $S D=3.65$ years) from the Stanford University community. Complete data were collected from 139 of the participants (data were lost from two participants due to equipment malfunction and two due to noncompliance). The experiment was performed in accordance with a protocol approved by the Stanford University IRB. All participants gave written informed consent and were remunerated $\$ 10 / \mathrm{hr}$.

\section{Procedure}

Overview Participants completed a set of questionnaires and performed four cognitive tasks (see Supplemental Materials for details). The questionnaires included the Media Multitasking Index (MMI; Ophir et al., 2009) and inventories for impulsivity and ADHD. The cognitive paradigms included two visual WM tasks and two recognition memory tests. All significant effects are reported.

Working memory task: Rectangles Participants first performed a standard visual WM task that required attentional filtering (Vogel, McCollough, \& Machizawa, 2005). Each trial consisted of an array of two target rectangles, colored red, along with $0,2,4$, or 6 distractor rectangles, colored blue (see Fig. 1a). Participants were instructed to first encode the orientations of the red rectangles - ignoring blue rectanglesduring the encoding period, then remember these orientations over the delay period, and finally detect whether either of the targets changed orientation between encoding and test. Participants indicated they detected a change (right index finger button press) or no change (right middle finger button press).

Working memory task: Objects Participants next performed a modified version of the visual WM task, wherein rectangles were replaced with common objects arranged in a circle (see Fig. 1b). Instructions were the same as in the rectangles task.

Recognition memory tasks Participants next performed (a) an old/new recognition memory test for target objects from the WM task, interspersed with new objects (see Fig. 1c) and then (b) a similar test for distractor objects from the WM task (see Fig. 1d). Participants responded with an old/ new judgment that included their confidence in the decision (high or low).

\section{Results}

\section{Questionnaires}

Media multitasking index Across all 139 participants, the median MMI score was 4.34 (mean $=4.41 \pm 1.91)$. We identified 36 HMMs (mean $=6.92 \pm 1.23$ ) and 36 LMMs (mean score $=2.19 \pm 0.70)$.

Impulsivity index The mean BIS-11 score was 61.38 $( \pm 10.57)$; HMMs did not significantly differ from LMMs across subscales, $F(1,201)=2.40, p=.12 ; \mathrm{HMM}_{\text {All scales }}=$ $62.79 \pm 10.81, \mathrm{LMM}_{\text {All scales }}=59.86 \pm 11.57$. 
A. WM: Rectangles task

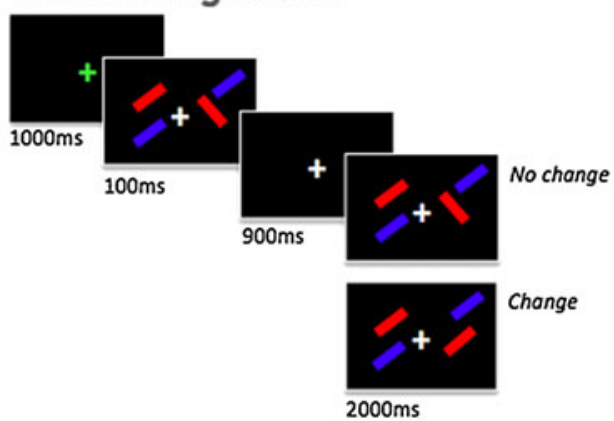

C. LTM: Target objects

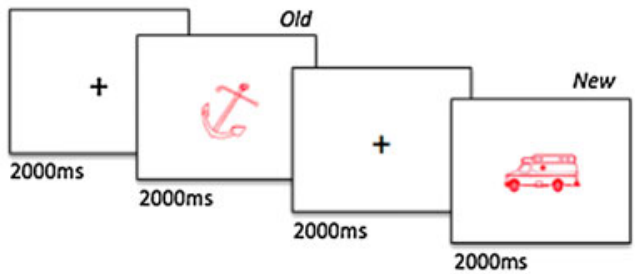

Fig. 1 Schematic of the working memory and long-term memory tasks. a. Participants first performed a standard version of a visual WM task that required attentional filtering at encoding (Vogel et al., 2005). Participants first viewed an array of colored rectangles (red and blue) and were instructed to attend to the red and ignore the blue rectangles. Two red (target) rectangles always appeared, along with $0,2,4$, or 6 blue (distracting) rectangles. Participants were instructed to detect whether either of the red (target) rectangles changed orientation from first to second presentation. b. The standard WM task was modified to include trial-unique common objects. Target and distractors $(0,2,4$, or 6$)$ could

ADHD index The mean ADHD score was $2.41( \pm 1.59)$; HMMs scored significantly higher than LMMs, $F(1,54)=$ 9.30, $p=.0033 ; \mathrm{HMM}=2.92 \pm 1.61, \mathrm{LMM}=1.97 \pm 1.65$.

Relationship between MMI, impulsivity, and ADHD Across all participants, MMI score positively correlated with ADHD, $\mathrm{r}_{136}=.30, p=.00036$, and impulsivity across subscales, $r_{136}=.17, p=.046$. The relationship between impulsivity and MMI was driven by the Attention subscale $(r=.24$, $p=.0046)$, with no significant effects in the other subscales (Motor: $r=.078, p=.36$; Nonplanning: $r=.065, p=.45$ ). The ADHD and overall impulsivity scores significantly correlated, $\mathrm{r}_{136}=.56, p=1.1 * 10^{-12}$.

\section{Working memory and long-term memory performance}

We first examined group effects (HMMs vs. LMMs) on performance and then, for effects of interest, we further tested whether performance continuously scaled with MMI score (i.e., across all participants).

Working memory: Rectangles We analyzed WM performance following Vogel et al. (2005): $K=S *(H-F)$, where

\section{B. WM: Common objects task}

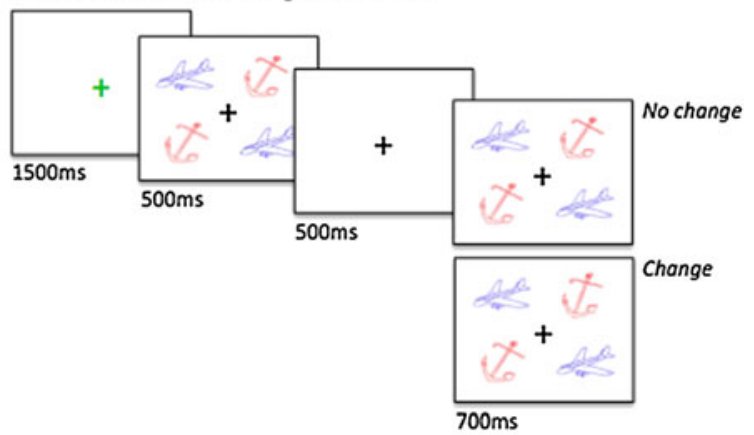

D. LTM: Distractor objects

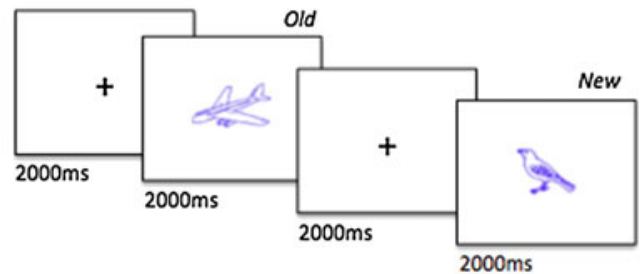

appear in any of 8 positions in a circular annulus around fixation (NB, size of objects depicted relative to frame is not representative). Participants were again instructed to detect whether either of the red objects changed orientation from first to second presentation. c. The ability to retrieve the target objects encountered in the $\mathrm{WM}_{\text {objects }}$ task was assessed by a recognition memory test, which interspersed objects that were targets in the $\mathrm{WM}_{\text {objects }}$ task with novel objects. $\mathbf{d}$. The ability to retrieve distractor objects encountered in the $\mathrm{WM}_{\text {objects }}$ task was assessed by a recognition memory test, which interspersed objects that were distractors in the $\mathrm{WM}_{\text {objects }}$ task with novel objects. (Color figure online.)

$K$ is WM capacity, $S$ the size of the target array (2), $H$ the proportion of correct changes detected (hit rate), and $F$ the proportion of changes incorrectly reported (false alarm rate). As measured by $K$, LMMs were able to hold more taskrelevant information in mind relative to HMMs (see Fig. 2a, left panel); Group (HMM, LMM) x Distractor Load (0, 2, 4, 6) ANOVA showed a main effect of Group: $F(1,256)=$ $4.88, p=.028$. This difference was driven by a greater tendency for HMMs to incorrectly endorse a change, when none occurred ("false alarms"; FAs), ANOVA on FA rate showed a main effect of Group: $F(1,256)=7.52, p=$ .0065 . Hit rate did not significantly differ across Groups: $\mathrm{F}(1,256)=1.27, p=.26$, and the Group $\mathrm{x}$ Hit/FA interaction was significant, $\mathrm{F}(1,548)=5.39, p=.021$.

We also interrogated the data in a signal detection theory (SDT) framework (Green \& Swets, 1966) to determine whether HMMs' reduced WM performance reflects (a) reduced discriminability to detect a change in the WM arrays, as measured by $d^{\prime}{ }_{\mathrm{WM}}\left(d^{\prime}=Z_{\mathrm{Hits}}-Z_{\mathrm{False}}\right.$ Alarms $)$, and/or (b) a different bias to report changes, as measured by $C_{\mathrm{WM}}\left(C=-1 / 2\left[\mathrm{Z}_{\mathrm{Hits}}+\mathrm{Z}_{\text {False Alarms }}\right]\right)$. Relative to LMMs, HMMs had a poorer ability to discriminate between the presence versus absence of change (see Fig. 2a, middle 


\section{A. Rectangles task}

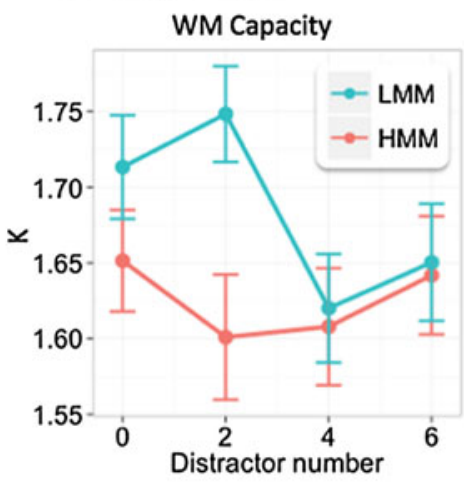

B. Common objects task

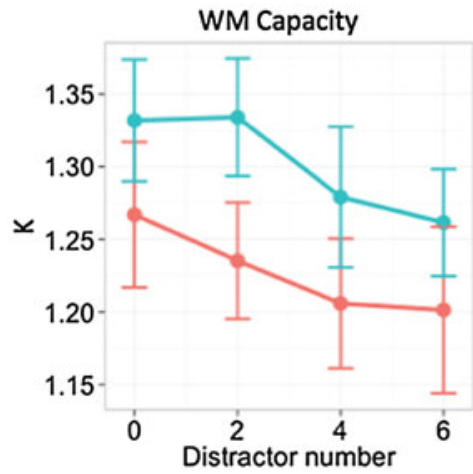

Working Memory
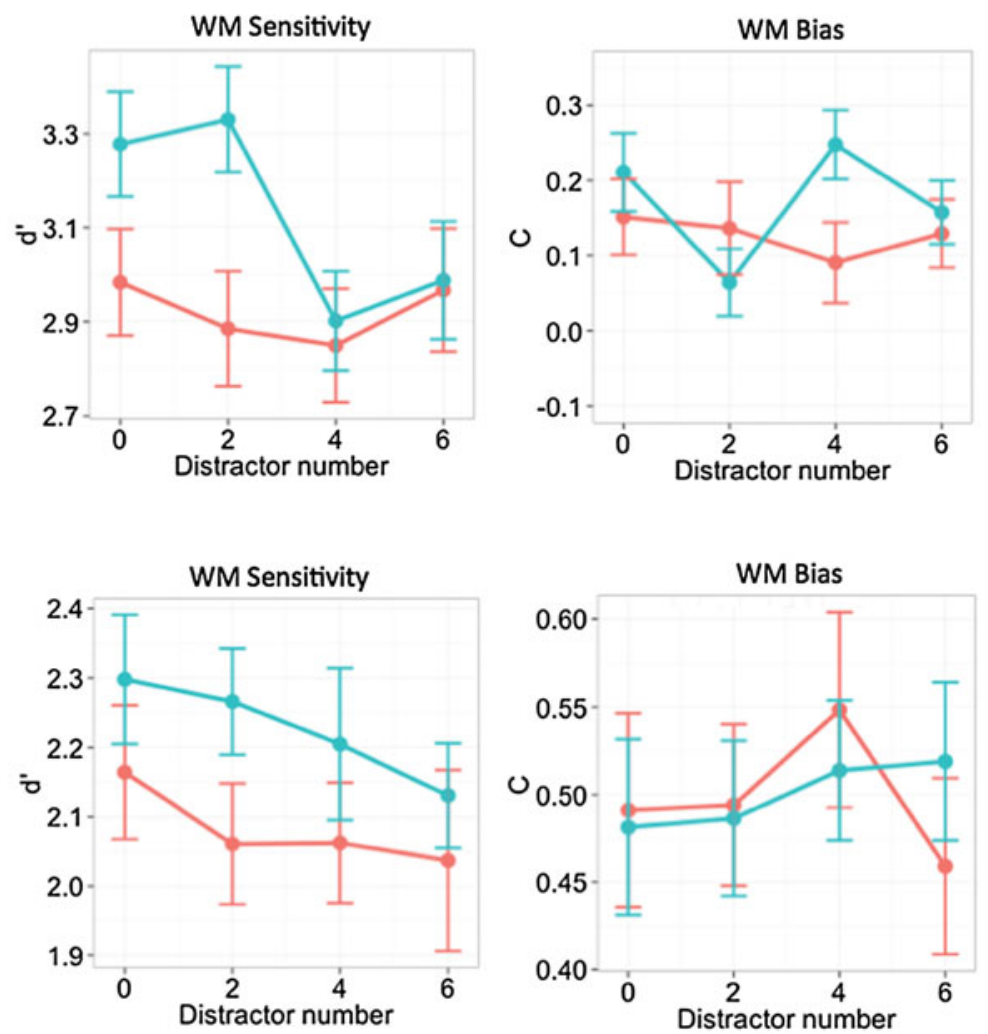

Fig. 2 Performance on the working memory tasks. a. Light media multitaskers (LMMs; blue) exhibited better working memory performance ( $K$; left panel) than heavy media multitaskers (HMMs; red). This was driven by better discriminability ( $d^{\prime}$; middle panel) to detect differences between the presence or absence of a change in orientation of the target rectangles, and not a more liberal decision bias

panel), $d^{\prime}$ WM by Group and Distractor Load; main effect of Group: $\mathrm{F}(1,256)=5.92, p=.016$. HMMs and LMMs did not differ in bias (see Fig. 2a, right panel), $C_{\mathrm{WM}}$ by Group and Distractor Load; main effect of Group: $F(1,256)=1.48, p=$ .23. Thus, reduced WM performance in people who frequently media multitask appears to be driven by discriminability differences: HMMs hold fewer or less precise representations of target information in WM.

To determine whether WM performance scales linearly across all levels of media multitasking, we regressed all 139 participants' MMI scores against their $d^{\prime}$ wM. This revealed a significant negative relationship: The higher the MMI score, the lower the WM discriminability, $d^{\prime}{ }_{\mathrm{WM}} \sim \mathrm{MMI}$, with Distractor Load as a factor (i.e., $d^{\prime}{ }_{\mathrm{WM}} \sim \mathrm{MMI} *$ Load): multiple regression $r=.16$; effect of MMI, $t=-2.61, p=.0092$. As was the case with group effects, the relationship between bias and MMI was not significant, $C_{\mathrm{WM}} \sim \mathrm{MMI} *$ Load: multiple regression $r=.098$; effect of MMI, $t=-1.10, p=.27$. Discriminability differences appeared to be due to FA rates and not hit rates: participants with higher MMI scores exhibited significantly higher FA rates, FA rate $\sim$ MMI * Load: multiple to endorse a change $(C$; right panel). b. This overall pattern was similar when the WM task required trial-unique objects to be held in mind: WM performance ( $K$; left panel) was better for LMMs than HMMs, and this performance was driven by discriminability $\left(d^{\prime}\right.$; middle panel) and not decision bias $(C$; right panel). (Color figure online.)

regression $r=.18$; effect of MMI, $t=2.97, p=.0032$, but not significantly lower hit rates, Hit rate $\sim$ MMI * Load: multiple regression $r=.094$; effect of MMI, $t=-1.20, p=.23$.

Working memory: Common objects A similar pattern of results was observed using the Objects variant of the WM task. Specifically, HMMs again exhibited significantly lower WM performance than LMMs (see Fig. 2b, left panel); $K$ by Group and Distractor Load, main effect of Group: $F(1,272)=5.45, p$ $=.020$, and this difference was due to a greater tendency to endorse a change when none occurred, FA rate by Group and Distractor Load, main effect of Group: $\mathrm{F}(1,272)=4.49, p=$ .035. Again, hit rate did not significantly differ across Groups (Hit rate by group and distractor load, main effect of Group: $F(1,272)=2.19, p=.14$. Finally, HMMs demonstrated reduced discrimination relative to LMMs (see Fig. 2b, middle panel); $d^{\prime}$ wM by Group and Distractor Load, main effect of Group: $F(1,272)=4.56, p=.034$, with no difference in bias (see Fig. 2b, right panel), $C_{\mathrm{WM}}$ by Group and Distractor Load, main effect of Group: $F(1,272)<1$. 
Across-participant regression revealed that while higher MMI scores numerically tended to be associated with lower WM discriminability, this relationship only trended toward significance, $d$ ' ${ }_{\mathrm{WM}} \sim \mathrm{MMI} *$ Distractor Load: multiple regression $r=.16$; effect of MMI, $t=-1.64, p=.10$. As in the rectangles task, this trend was associated with a slightly greater tendency to endorse a change when none occurred, although this relationship again only trended toward significance, FA rate $\sim$ MMI $*$ Load: multiple regression $r=.16$; effect of MMI, $t=1.64, p=.10$. Finally, MMI again did not correlate across participants with hit rate, Hit rate $\sim$ MMI * Load: multiple regression $r=.15$; effect of MMI, $t=-.87, p=.39$, or bias, $C_{\mathrm{WM}} \sim \mathrm{MMI} *$ Load: multiple regression $r=.099$, effect of MMI $t=-.42, p=.68$.

Taken together, these two WM studies indicate thatregardless of the nature of the information (common objects or rectangles) - HMMs demonstrate a deficit in WM that reflects a reduction in the number or precision of task-relevant representations that they can encode and/or maintain in WM.

Long-term memory: Target objects Paralleling the effects observed in WM, HMMs, relative to LMMs, exhibited reduced LTM performance, manifested as a reduced ability to discriminate the previously encountered WM targets from novel objects (see Fig. 3a, left panel); $d_{\text {LTM }}$ by Group, Distractor Load, and Confidence (high vs. low), main effect of Group: $F(1,532)=9.39, p=.0023$. Here HMMs' poorer discrimination was accompanied by a more liberal decision bias when looking across all trials, with HMMs demonstrating a stronger bias to endorse objects as recognized, $C_{\mathrm{LTM}}$ by Group, Distractor Load, and Confidence, main effect of Group: $F(1,532)=5.83, p=.016$. However, when confined to high confidence responses only, HMMs and LMMs were equally conservative, $F(1,267)=1.31, p$ $=.25$. Across participants, higher MMI scores correlated with reduced LTM performance, $d_{\text {LTM }} \sim$ MMI * Distractor Load * Confidence: multiple regression $r=.65$; effect of MMI, $t=-2.67, \mathrm{p}=.008$, even when confined to high confidence retrieval responses, multiple regression $r=.16$; effect of MMI, $t=-2.47, p=.014$.

To test whether WM performance - using the standard $K$ metric - predicted LTM performance, we regressed all participants' LTM discrimination scores ( $\left.d^{\prime}{ }_{\text {LTM }}\right)$ onto their performance on the WM objects task. There was a significant positive relationship between the ability to hold objects in WM and the ability to later recognize those previously encountered objects (NB, this pattern was significant when LTM performance was assessed collapsed across decision confidence), $r_{136}=.31$, $p=2.3 * 10^{-4}$, as well as when restricted to high confidence decisions, $r_{136}=.33, p=8.6 * 10^{-5}$; thus, we report high confidence outcomes henceforth (see Fig. 3a, right panel, green).

This relationship between the ability to encode and maintain common objects in WM and the ability to later retrieve those objects from LTM is important, and yet does not adjudicate between alternative hypotheses about whether impaired WM acts to reduce (a) the encoding of information into LTM, or (b) task performance more generally, perhaps by reducing the ability to hold information online during LTM tasks. A first step toward adjudicating between these alternatives may come from assessing whether WM performance predicts LTM performance for completely different information. Here, we tested this hypothesis by determining whether WM performance on the rectangles task predicted LTM performance (for the objects), and found that the predictive relationship held (see Fig. 3a, right panel, orange); $r_{132}=.22, p=.0093$. Because WM performance for the two types of material was correlated, we further examined whether performance on the rectangles task provided predictive information about LTM above and beyond that which was provided by the objects task. A multiple regression analysis revealed a strong predictive relationship, even after removing variance associated with the WM objects task, multiple regression $r=.29$; effect of $K_{\text {rectangles }}, t=3.52, p=.00046$, suggesting that WM performance may have a more general impact on LTM.

Taken together, the foregoing results show that people who frequently engage with multiple media streams during their daily lives demonstrate worse LTM for previously encountered target information. Importantly, HMMs' diminished LTM and WM performance occurred for information that was encountered while the participants were ostensibly single-tasking.

Long-term memory: Distractor objects A final question concerned the LTM fate of distractor objects encountered during the WM objects task, as the answer may shed light on mechanisms underlying how HMMs manage competing representations in the WM task. We predicted two possible scenarios: (1) at encoding, HMMs attend to distractor objects at the expense of target objects, resulting in better representation of distractor objects in WM for HMM vs. LMMs, and ultimately leading to better LTM of distractor objects for HMMs, or (2) the ability to interrogate representations held in mind, whether during WM or LTM tasks, is reduced in HMMs, manifesting as worse LTM performance in HMMs than LMMs, for both targets and distractor objects.

An ANOVA of distractor LTM performance revealed a trend favoring the second scenario, in that HMMs remembered the distractors more poorly than LMMs (see Fig. 3b, left panel); $d_{\text {LTM }}$ by Distractor Load $(2,4,6)$, Group, and Confidence: main effect of Group: $F(1,399)=3.47, p=.063$. Interestingly, the number of times a distractor was displayed in the array (i.e., Distractor Load) had no effect on LTM for distractors, $\mathrm{F}(1,399)<1$.

We next examined whether WM performance predicts LTM performance for the distractor objects (as it did for target objects). To do so, we regressed all participants' ability to retrieve distractors from LTM $\left(d_{\text {LTM }}\right)$ onto their 


\section{A. Target objects}

LTM Performance

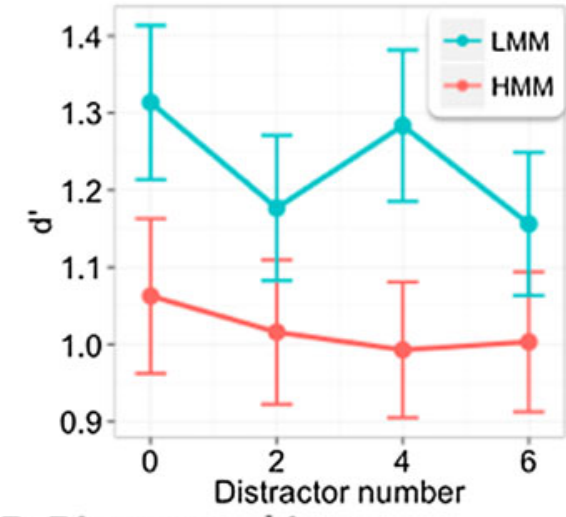

B. Distractor objects

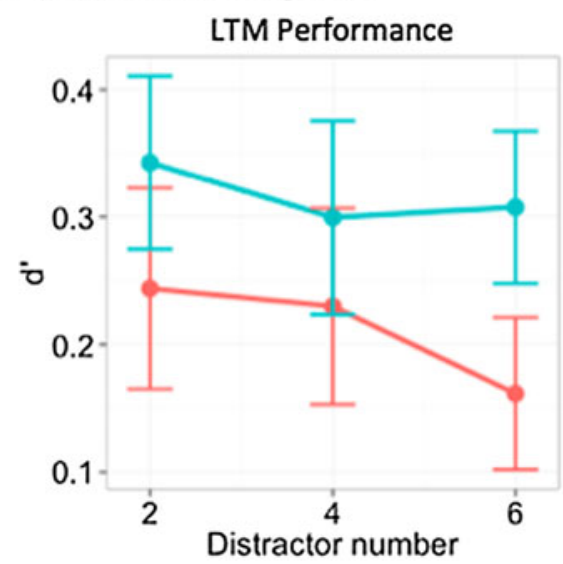

Fig. 3 Performance on the long-term memory tasks, for target and distractor objects encountered in WM objects task. a. Target objects encountered in the $\mathrm{WM}_{\text {objects }}$ task were better remembered by LMMs than HMMs (left panel), and, across participants, $\mathrm{WM}_{\text {object }}$ performance predicted later LTM for the target objects (right panel). b. Distractor

performance in the WM objects task ( $K$, the index of how well target information was held in mind). We found a positive relationship between $\mathrm{K}$ and the ability to later confidently recognize distractor objects (see Fig. 3b, right panel, green); $K_{\text {objects }} \sim d^{\prime}$ LTM-distractors ${ }^{*}$ Confidence: multiple regression $r=.72$, effect of $K_{\text {objects, }} t=4.48, p=1.1$ $* 10^{-5}$. This relationship was similar across WM tasks, with WM performance in the rectangles task also predicting long-term memory for distractor objects (see Fig. $3 b$, right panel, orange); $K_{\text {rectangles }} \sim d^{\prime}$ LTM-distractors $*$ Confidence: multiple regression $r=.33$, effect of $K_{\text {rectangles }}$ $t=2.11, p=.036$, although the relationship was not significant after removing variance associated with WM performance for the objects task, likely due to floor effects, multiple regression $r=.32$; effect of $K_{\text {rectangles, }} t<1$.

Together, these findings show that WM performance in general-across different tasks (rectangles/objects) and different
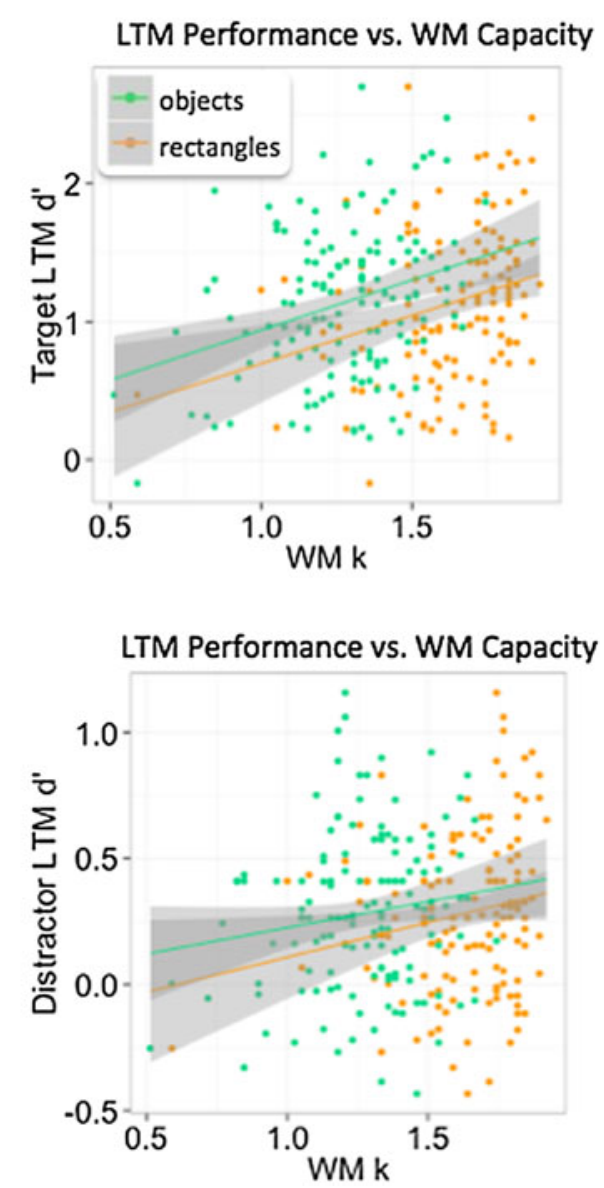

objects encountered in the $\mathrm{WM}_{\text {objects }}$ task were also better remembered by LMMs than HMMs (though they were poorly remembered, overall, by both groups; left panel), and, across participants, $\mathrm{WM}_{\text {object }}$ performance predicted later LTM for the distractor objects (right panel). (Color figure online.)

information (target/distractor objects) - predicts LTM performance, suggesting that WM deficits are likely exerting their effects at both encoding and retrieval.

Relationship between task performance and impulsivity Given the observed relationship between impulsivity and MMI score-driven by the Attentional Impulsivity subscale - we examined whether this subscale predicted task performance ( $d$ ' and $C$ in WM and LTM tasks). Across all participants, the Attentional subscale negatively predicted $d^{\prime}$ in both WM tasks, rectangles: attentional impulsivity $\sim d^{\prime}{ }_{\mathrm{WM}}$ * Load: multiple regression $r=.14$, effect of $d^{\prime}, t=-2.15, p=$ .032 ; objects: multiple regression $r=.15$, effect of $d^{\prime}, t=-$ $2.75, p=.0062$, but did not show a relationship with d' in the LTM task $(p>.6)$ or with $C$ in any task (all $p$ s $>.05)$. Thus, higher self-reported attentional impulsivity was associated with worse discrimination in both WM tasks. 


\section{Discussion}

The study yielded four important findings. First, in two independent tasks, HMMs showed reduced WM performance regardless of whether external distractors were present or absent. This performance decline was evident in reduced $K$ and $d$ ' measures of WM ability. Moreover, when media multitasking was treated as a continuous variable, a negative relationship between chronic media multitasking behavior and WM performance was observed. Second, there was a coupling between WM and LTM, with LTM performance predicted by WM abilities more broadly (across different WM tasks and different content). This pattern suggests that WM deficits likely exert effects on LTM at both encoding and retrieval rather than selectively reducing the fidelity of the representations encoded into LTM. Third, the observation that discriminability and not decision bias accounted for differences in WM and LTM performance suggests that HMMs' reduced amount or precision of information held in mind - whether during WM or LTM tasks - drives performance differences. Finally, in contrast to our predictions, the higher impulsivity of HMMs correlated with their reduced WM discrimination but not with a tendency to require less evidence to reach a decision.

A small but growing number of studies have investigated task performance of heavy and light media multitaskers or have correlated MMI score with task performance, revealing various behavioral differences. For instance, HMMs were observed to have difficulty (a) filtering distracting information, whether the information came from the environment (external distraction) or from memory (internal distraction; Ophir et al., 2009), and (b) ignoring attention-capturing information, regardless of whether or not they were instructed to ignore the information (Cain \& Mitroff, 2011). HMMs were observed to adopt a split visuospatial attention mode (the allocation of attention to multiple locations), whereas LMMs adopt a more unitary mode (Yap \& Lim, 2013), and individuals with higher media multitasking scores exhibit enhanced multisensory integration (Lui \& Wong, 2012). Other studies investigating task-switching abilities have reported equivocal results, showing that, relative to LMMs, HMMs were worse (Ophir et al., 2009; Sanbonmatsu et al., 2013), better (Alzahabi \& Becker, 2013), or equivalent (Alzahabi \& Becker, 2013; Minear et al., 2013).

One mechanism proposed to underlie the differences associated with chronic media multitasking is that HMMs exhibit a broader attentional scope (Cain \& Mitroff, 2011; Lui \& Wong, 2012; Ophir et al., 2009). A wider scope may change the manner in which available information is filtered in order to optimize task goals, manifesting as attention to both goalrelevant and goal-irrelevant information. As a consequence, goal-irrelevant information may compete with goal-relevant information, reducing task performance.
Here, a wider attentional bias at encoding (i.e., WM objects task) would result in competing WM representations of targets and distractors, giving rise to lower fidelity LTM representations of both, as was observed. However, the amount of external distraction present during WM did not differentially affect HMMs (c.f., Ophir et al., 2009), which suggests that their lower performance may be a result of continual distraction by information not under experimental control. Additionally, the present data suggest that lower fidelity encoding is not the only mechanism contributing to HMMs' poor LTM performance: that performance on an entirely different WM task (WM rectangles) predicted LTM for both targets and distractors suggests that HMMs exhibit a generalized reduction in the ability to hold or interrogate precise representations in mind, whether during WM or LTM tasks. Thus, the pattern of findings suggest that HMMs' reduced discrimination in WM and LTM may be a result of a wider attentional scope at both encoding and retrieval, allowing task-irrelevant information to continually compete with task-relevant information. This wide scope first serves to reduce the amount or precision of goal-relevant information held in mind and therefore encoded into LTM; during the subsequent retrieval from LTM, the wider attentional scope may result in the intrusion of task-irrelevant information, further degrading the ability to make accurate retrieval decisions.

Further bolstering the idea that a wider attentional scope impacts cognition at both encoding and retrieval is the finding that LTM for study distractors was worse, rather than better, for HMMs. To the extent that a wider attentional scope at encoding allowed more distractor information into WM for HMMs, distractors could have been better encoded by HMMs than by LMMs, which should have then led to better distractor memory. Instead, here we found distractor memory to be slightly worse in HMMs, suggesting that the seemingly wider attentional scope of HMMs has an impact on task performance more generally. It will be important in future investigations to determine just how extensively WM deficits impact cognition in HMMs.

Our findings additionally revealed that attentional impulsivity positively related to the degree to which participants multitasked with media. The BIS-Attention subscale has been shown to index self-reported factors of attention ("focusing on the task at hand") and cognitive instability ("thought insertions and racing thoughts"; Patton et al. 1995). These factors may describe well the phenomenology associated with adopting a broad attentional scope/reduced filter (see Supplement for further discussion).

In conclusion, the present findings point to a parsimonious and mechanistic explanation for many of the performance differences observed in the growing literature investigating chronic media multitaskers. That chronic media multitasking is associated with deficits in cognitive abilities that are critical for successful navigation through life-including holding 
information in mind and retrieving information from memory - calls for systematic investigations into what is cause and what is effect. Our increasingly media-saturated world may be nudging us toward an increasingly wider scope of attention, in which case how we choose to interact with media may significantly impact cognitive performance. On the other hand, adopting healthy media hygiene may make no difference if one's media multitasking behavior is due to a cognitive predisposition (e.g., impulsivity) that leads to, rather than is caused by, such multitasking. The relationship between media multitasking and academic outcomes also remains unknown, in college-age adults, as well as in younger students. Given the increasing understanding of the importance of WM and LTM to academic achievement, future studies should aim to determine whether and how media multitasking behavior relates to academic outcomes. Poorer WM and LTM could give rise to reduced classroom-based learning and testing performance. By contrast, there may be instances where the cognition associated with HMM behavior gives rise to superior academic outcomes. For example, if a broader attentional scope allows for reinstatement of related memories (e.g., Kuhl et al. 2011; Shohamy \& Wagner, 2008), this may support the generation of cognitive schemas that facilitate learning of academic content. Recommendations for parents, educators, students, and policymakers will depend on understanding the direction of causality between media multitasking and cognitive differences in students as well as in the general population.

Acknowledgements This work was supported by NIMH grant R21-MH099812.

Conflict of Interest The authors declare no competing financial interests.

\section{References}

Alzahabi, R., \& Becker, M. W. (2013). The association between media multitasking, task-switching, and dual-task performance. Journal of
Experimental Psychology-Human Perception and Performance. doi:10.1037/a0031208

Cain, M. S., \& Mitroff, S. (2011). Distractor filtering in media multitaskers. Perception, 40, 1183-1192. doi:10.1068/p7017

Green, D. M., \& Swets, J. A. (1966). Signal detection theory and psychophysics. New York, NY: Wiley.

Kuhl, B., Rissman, J., Chun, M., \& Wagner, A. (2011). Fidelity of neural reactivation reveals competition between memories. Proceedings of the National Academy of Sciences of the United States of America, 108(14), 5903-5908. doi:10.1073/pnas. 1016939108

Lui, K. F. H., \& Wong, A. C. N. (2012). Does media multitasking always hurt? A positive correlation between multitasking and multisensory integration. Psychonomic Bulletin and Review, 19(4), 647-653. doi: 10.3758/s13423-012-0245-7

Minear, M., Brasher, F., McCurdy, M., Lewis, J., \& Younggren, A. (2013). Working memory, fluid intelligence, and impulsiveness in heavy media multitaskers. Psychonomic Bulletin and Review, 20(6), 1274-1281. doi:10.3758/s13423-013-0456-6

Ophir, E., Nass, C., \& Wagner, A. D. (2009). Cognitive control in media multitaskers. Proceedings of the National Academy of Sciences of the United States of America, 106(37), 15583-15587. doi:10.1073/ pnas.0903620106

Patton, J. H., Stanford, M. S., \& Barratt, E. S. (1995). Factor structure of the Barratt impulsiveness scale. Journal of Clinical Psychology, 51(6), 768-774.

Rideout, V. J., Foehr, U. G., \& Roberts, D. F. (2010). Generation M2: Media in the lives of 8-to 18-year-olds. Oakland, CA: Henry J. Kaiser Family Foundation.

Sanbonmatsu, D. M., Strayer, D. L., Medeiros-Ward, N., \& Watson, J. M. (2013). Who multi-tasks and why? Multi-tasking ability, perceived multi-tasking ability, impulsivity, and sensation seeking. PLOS ONE, 8(1), e54402. doi:10.1371/journal.pone.0054402

Shih, S.-I. (2013). A null relationship between media multitasking and well-being. PLoS ONE, 8(5), e64508. doi:10.1371/journal.pone. 0064508

Shohamy, D., \& Wagner, A. D. (2008). Integrating memories in the human brain: hippocampal-midbrain encoding of overlapping events. Neuron, 60(2), 378-389. doi:10.1016/j.neuron.2008.09.023

Vogel, E. K., McCollough, A. W., \& Machizawa, M. G. (2005). Neural measures reveal individual differences in controlling access to working memory. Nature, 438(7067), 500-503. doi:10.1038/ nature 04171

Yap, J. Y., \& Lim, S. W. H. (2013). Media multitasking predicts unitary versus splitting visual focal attention. Journal of Cognitive Psychology, 25(7), 889-902. doi:10.1080/ 20445911.2013.835315 